\title{
Emergency Detection and Monitoring Daily Routine of Cattle using loT
}

\author{
Vijayashree B. R. \\ Department of ECE \\ Sambhram Institute of \\ Technology \\ Bangalore-97
}

\author{
Sheetal C. Medhavi \\ Department of ECE, \\ Sambhram Institute of Technology \\ Bangalore-97
}

\author{
Simran \\ Department of ECE \\ Sambhram Institute of \\ Technology \\ Bangalore-97
}

\author{
Sunilkumar R. \\ Department of ECE \\ Sambhram Institute of \\ Technology \\ Bangalore-97
}

\author{
K. Ezhilarasan \\ Department of ECE, \\ Sambhram Institute of Technology \\ Bangalore-97
}

\begin{abstract}
Todays life human can't imagine their life without technology. The era has modified and developed many projects such as advanced cattle health monitoring system using pic microcontroller and IOT. The system collects $\mathrm{x}, \mathrm{y}, \mathrm{z}$ axis acceleration data through triaxial accelerometer. The intention of this research is to establish a relationship between dairy cattle diseases with various non-invasive sensors for the development of a health monitoring system. Based on cattle health monitoring system critical parameters affecting cattle health which includes body temperature, resperation, humidity ,heartbeat and rumination continuously monitored. ESP8266 $\mathrm{Wi}-\mathrm{Fi}$ module is used as transceiver. LM-35 is used temperature sensor to monitor the body temperature of the cattle. Two number of ADXL335 accelerometer sensors are used in this wearable device for lameness detection and alerting during pregnancy. MQ-02 smoke sensor is used for detection of smoke level in cattle farm environment. InfraRed sensor is used for count the occupancy level of cattle in farm for smart lighting and smart ventilation purpose.
\end{abstract}

\section{Keywords}

IOT,PICMicrocontroller,ESP-Wifimodule,sensors, 3-axial acceleration.

\section{INTRODUCTION}

Now a day's food features is not only decided by the overall environment and security of the ending product but also by the animal's welfares status by which the food is produced. When we will develop the animal's health on that time it will affect the quality of product, pathology and safety [7]. The financial and common activities of human culture is very important in developing countries where many people depends on livestock based activities and this livestock production will stay for many years. The interest in monitoring cow's behavior for automated detecting estrus or unhealthy motion has steadily grown in recent years. In particular, large-scale dairy operations require an efficient automated heat detection system to accurately determine when the cows are ready for insemination. Accurate detection of estrus behavior for breeding on dairy farms is essential to dairy profitability as missed estrus periods cost dairymen. Many technologies have been attempted to detect estrus behavior in cattle with varying success. However, not all of commercial devices developed for automated heat detection and wireless data transmission provide required information such as activity level, traveling rate, and distance traveled of a cow. A smoke detector or smoke alarm is a device that detects smoke and issues an alarm to alert nearby people that there is a potential fire. Smoke detectors can work by optical detection (photoelectric) or by physical process (ionization), but some smoke detectors also utilize both types of detector to increase the sensitivity to smoke. In this paper, enhancement done on already available photoelectric smoke detector circuitry by integrating some distinct features like wireless technology, SMS sending through GSM module and fire extinguishing vehicle [4]. This features increase the safety and reliability of the smoke and fire detection technology.

\section{LITERATURE SURVEY}

[1]Amruta Helwatkar et.al , [Anshul Awasthi, Daniel Riordan and Joseph Walsh] proposed non-invasive sensor technology for the development of diary cattle health monitoring system. this research is to establish a relationship between dairy cattle diseases with various non-invasive sensors for the development of a health monitoring system. The aim is to consider the nature of the diseases a cow may have and relate it with one or many sensors that are suitable for accurate measurement of the behavioral changes. This proposed research uses ontological relationship mapping or ontology matching to integrate heterogeneous databases of diseases and sensors.

[2]Toufiqul Islam, Syed Asif Abdullah, and Golam Sarowar proposed enhanced wireless control system for smoke and fire detection.It describes the design and engineering of a wireless control system for smoke and fire detection with alarming provision, sms sending and fire extinguishing by a vehicle. It make use of PIC microcontroller for the controlling of the system.

[3]David Hanson and Changki Mo proposed monitoring cattle motion using 3-axil acceleration and GPS data. This paper examines feasibility of a cattle monitoring device that can sense, record, and wirelessly transmit the 3 -axis acceleration and global positioning system (GPS) data of the cow for accurate and timely monitoring. The device is intended to use for estrus detection by interpreting the measured data to identify cow's behavior and act. This proposed research is to automate estrus detection for artificial insemination, but the capability to track the cow's motion characteristics may provide dairy operators with the capacity to assess the cow's behavior and act to improve the overall well-being and health of the cow. It makes use of GPS. 
[4] Atef Agarwal1 et.al, [ Esha Mehandiratta2, Rohit Sanket3, Rohit Samkaria4, Abuja's Shah5, Rajesh Singh6 and Anita Gehlot7] proposed WPAN based cattle health monitoring with LabVIEW as a data logger. A wireless cattle health monitoring system to measure health and psychological parameter of cattle has been developed using environmental temperature and humidity, body temperature, rumination, heartbeat, and sweat sensors. The system developed is capable to analyze stress level of cattle with reference to THI (Thermal Humidity Index). The ATmega16 controller and ZigBee has been used to develop the wireless sensor and receiver nodes. LabVIEW is used as a real time data logger for monitoring the output of various sensors over the PC.

[5]Ans elemi B.Lukonge et.al, [Dr.Shubi Kaijage, Ramadhani S. Sinde] proposed review of cattle monitoring system using wireless network. The cattle industry is an integral part of the world economy. The continued production of quality beef requires new and improved methods for long term monitoring of animal health. Additional benefits can be realized from this class of technology, such as the ability to identify the presence of disease early and thereby prevent its spread. An important element of health assessment is the ability to monitor vital data such as core body temperature. It make use of wireless sensor network and GPS

[6] Snehal. S Kharde proposed Advance Cattle Health Monitoring System Using Arduino and IOT In the Wireless Sensor Based cattle health monitoring system, critical parameters affecting cattle health which includes body temperature, respiration, humidity, heart beat and rumination are continuously monitored. In this framework, Arduino UNO microcontroller is utilized to sense the various activities of animals like body temperature, respiration, humidity, heart beat and rumination. ESP8266 Wi-Fi module is used as transceiver. The i-Chart app is used to display the graph.

[7]Ankit R. Bhavsar1, Disha J. Shah2 and Harshal A. Arolkar3 proposed Distributed Data Storage Model for Cattle Health Monitoring Using WSN Wireless Sensor Networks (WSNs) are widely used for monitoring physical happenings of the environment. The data gathered using WSN is bulky, heterogeneous and distributed through the network. As the data would be massive, the data gathering process needs appropriate data storage. In WSN, three data storage and retrieval methods namely External Storage (ES), Local Storage (LS) and Data Centric Storage (DCS) are generally used. The external storage is used when the data is to be stored on an external storage device. Here, the nodes send data to the base station without any query being generated by the user; also it does not perform any kind of aggregation. This results in high traffic that causes unbalanced energy consumption and delayed services. The local storage has an inbuilt database that keeps the data locally. The node is unaware of the target node to which the data is to be transmitted.

[8]Parkash, Prabu V, Dandu Rajendra proposed Internet of Things Based Intelligent Street Lighting System for Smart City. Currently we have a manual system where the street lights will be switched $\mathrm{ON}$ in the evening before the sunsets and they are switched OFF in the next day morning after there is sufficient light on the outside[1]. But the actual timing for these lights to be switched $\mathrm{ON}$ is when there is absolute darkness. With this, the power will be wasted up to some extent. This project gives solution for electrical power wastage also the manual operation of the lighting system is completely eliminated. The proposed system provide a solution for energy saving. This is achieved by sensing and approaching a vehicle using an IR transmitter and IR Receiver couple. Upon sensing the movement the sensor transmit the data to the microcontroller which furthermore the Light to switch ON .Similarly as soon as the vehicle or an obstacle goes away the Light gets switched OFF as the sensor sense any object at the same time the status(ON/OFF) of the street light can be accessed from anywhere and anytime through internet.

[9] Yogita Patil, Ramandeep Singh proposed Smart Water Tank Management System for Residential Colonies Using Atmega128A Microcontroller. For this project, microcontroller has been used, a reservoir, water tank and water pump. Water pump has been controlled using relay and contact sensor. Six homemade contact sensors are used (three for each tank) are used to detect water level.

\section{PROPOSED MODULE}

The Advanced cattle health monitoring system is divided in to three main units as shown in the Figure 1 that interrelate with each other to provide actual period observing, processing and recording. They are data gaining unit, Data administering unit and Data interact unit. Data gaining unit mainly consist of body temperature sensor, respiration sensor, humidity sensor, heart beat and rumination sensor with interfacing to PIC microcontroller. The data gaining unit obtain the data and makes it accessible for the data administering and data interact unit. The sensors are allowed for simple and general automatic measurement of numerous health factors. Such health sensors will be mounted on the cattle body, which continuously observe the body issues of the cattle like body temperature, respiration, humidity etc. and delivers output in the type of electrical signs. These signs are then compared to a standard limit of normal values set as the starting point in the data administering unit. The main function of this unit is when the animal will suffer from disease people use to take that animal to the doctor for diagnoses but sometimes doctors will not be available in hospitals so using this advance monitoring system we can sense the various activities of animals like body temperature, respiration, humidity etc. and we can send the animal health graph to the doctor mobile using ESP8266 WIFI module. It will reduces the human effort . It will save the cattle life. Improve the quality of output. It will continuously monitor the cattle health parameter. We can use this system in poultry farms. We can use this system in Cow farms. We can use this system in Sheep farm. .We can use this system in Sericulture farm. 


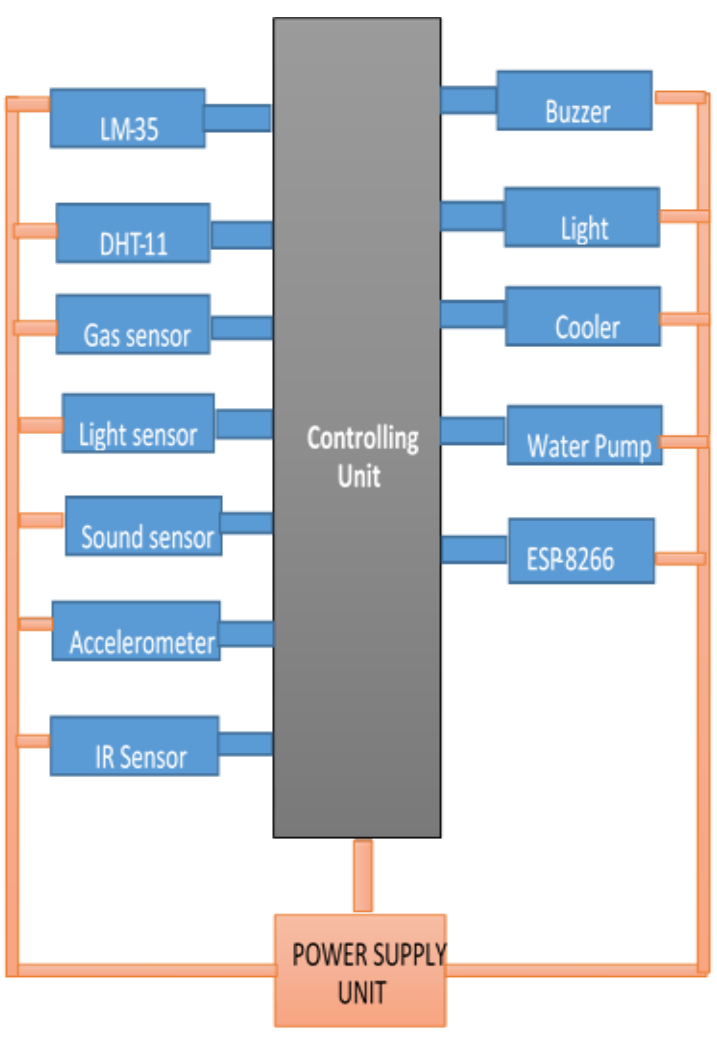

Figure 1: Overview of proposed module

\section{CONCLUSION}

Proposed system will provide the farmers with good efficiency. The farm is fully protected. The microcontroller will control the overall process taking place in the farm. The cattle body temperature and moisture level is sensed with the help of sensors and the values are displayed with the help of an LCD display. The overall process is under the control of microcontroller. We can expect total automation in cattle farm and it will leads to increasing the productivity. It is a wearable hardware gadget which will monitor the cattle's health parameters and it will take some necessary action in some critical conditions. We will get all the data from website for further analysis. Smart ventilation system improves cattle health by preventing environment to reach critical. Smart ventilation and smart lighting saves consumption of electricity which leads to save a lot of money. This system saves time and maintenance costs for the farmer ensuring improved cattle health and high yield.

\section{REFERENCES}

[1] Amruta Helwatkar, Daniel Riordan, and Joseph Walsh,"Sensor Technology For Animal Health Monitoring," Proceedings of the 8th International Conference on Sensing echnology, pp. 266-271, Sep. 2014.
[2]Toufiqul Islam, Syed Asif Abdullah, and Golam Sarowar, "Enhanced Wireless Control System for Smoke and Fire Detection," International Journal of Computer and Electrical Engineering, vol. 5, no. 2, pp. 233-236, Apr. 2013.

[3] David Hanson and Changki Mo, "Monitoring Cattle Motion using 3-axis Acceleration and GPS Data," Journal of Research in Agriculture and Animal Science, vol. 2, no. 10, pp. 01-08, Dec. 2014.

[4] Ateev Agarwal et al., "WPAN Based Cattle Health Monitoring With Labview as A Data Logger," International Journal of Future Generation Communication and Networking, vol. 9, no. 6, pp. 275284, 2016.

[5] Anselemi B. Lukonge, Dr. Shubi Kaijage, and Ramadhani S. Sinde, "REVIEW OF CATTLE MONITORING SYSTEM USING WIRELESS NETWORK," International Journal Of Engineering And Computer Science, vol. 3, no. 5, pp. 5819-5822, May 2014.

[6] M. Snehal. S. Kharde, "Advance Cattle Health Monitoring System Using Arduino and IOT," International Journal of Advanced Research in Electrical, Electronics and Instrumentation Engineering, vol. 5, no. 4, pp. 3365-3370, Apr. 2016

[7] Ankit R. Bhavsar, Disha J. Shah, and Harshal A. Arolkar, "Distributed Data Storage Model for Cattle Health Monitoring Using WSN,"Advances in Computer Science: an International Journal, vol. 2, no. 2, pp. 19 24, May 2013.

[8] Parkash, Prabu V, and Dandu Rajendra, "Internet of Things Based Intelligent Street Lighting System for SmartCity," International Journal of Innovative Research in Science, Engineering and Technology, vol. 5, no. 5, pp. 7684-7691, May 2016.

[9] Yogita Patil and Ramandeep Singh, "Smart Water Tank Management System for Residential Colonies Using Atmega128A Microcontroller," International Journal of Scientific \& Engineering Research, vol. 5, no. 6, pp. 355-357, Jun. 2014.

10] A. H. H. Nograles and F. S. Caluyo, "Wireless system for pregnancy detection in cows by monitoring temperature changes in body," Signal Processing and its Applications (CSPA), 2013 IEEE 9th International Colloquium on, Kuala Lumpur, 2013, pp. 11-16.

[11] Weizheng Shen, Congcong Chen, Shuang Zheng, Shanjun $\mathrm{He}$, and Mingda Li, "The Design of System about Cow Activity Based on SVM," International Journal of Smart Home, vol. 9, no. 3, pp. 91-100, 2015. 